\title{
Quantitative retrieval of biogeophysical characteristics using imaging spectroscopy - a mountain forest case study
}

\author{
M. E. Schaepman ${ }^{1}$, B. Koetz ${ }^{2}$, G. Schaepman-Strub ${ }^{2,3}$, N. E. Zimmermann ${ }^{4}$ \\ and K. I. Itten ${ }^{2}$ \\ ${ }^{1}$ Corresponding author. Centre for Geo-Information, Wageningen University and Research Centre, \\ Droevendaalsesteeg 3, NL-6708 PB Wageningen, The Netherlands, E-mail: Michael.Schaepman@wur.nl \\ Fax: +4116356846. \\ ${ }^{2}$ Remote Sensing Laboratories, Department of Geography, University of Zurich, Winterthurerstrasse 190, \\ CH-8057 Zurich, Switzerland \\ ${ }^{3}$ Nature Conservation and Plant Ecology, Wageningen University and Research Centre, Bornsesteeg 69, \\ NL-6708 PD Wageningen, The Netherlands \\ ${ }^{4}$ WSL, Swiss Federal Research InstitutL, Zürcherstrasse 111, CH-8903 Birmensdorf, Switzerland
}

Keywords: Biogeophysical and biogeochemical parameters, Earth observation, Imaging spectroscopy, Radiative transfer, Remote sensing.

\begin{abstract}
Quantitative remote sensing is becoming increasingly important due to the broader availability of well-calibrated airborne and spaceborne imaging spectrometers. We demonstrate the additional benefit of imaging spectrometers in the retrieval of biochemical and -biophysical variables using a general overview of recent contributions of these instruments in a programmatic context and describe in detail in a regional test case the inversion of a radiative transfer model to achieve the overall goal. Imaging spectroscopy today enables the biogeophysical and biogeochemical variables of the continental biosphere processes to be measured with calibrated physical units and quantifiable accuracy. During a large field experiment in the Swiss National Park in 2002, DAIS 7915 and ROSIS imaging spectrometer flights were carried out simultaneously with ground measurements. We constrain the inversion of a radiative transfer model (GeoSAIL) to retrieve parameters such as LAI, fractional cover, dry matter, and water content. We demonstrate that the retrievals are performed with high accuracy and conclude that the derived canopy variables represent the actual spatial distribution of forest biophysical and biochemical properties as they occur in the landscape.
\end{abstract}

Abbreviations: DAIS - Digital Airborne Imaging Spectrometer; LAI - Leaf Area Index; LUT - Lookup Table; NIR - Near Infrared part of the spectrum; RMSE - Root Mean Square Error; RTM - Radiative Transfer Model(s); SWIR - Shortwave Infrared part of the spectrum; VIS - Visible part of the spectrum.

Nomenclature: Nicodemus et al. (1977) modified by Martonchik et al. (2000) and appended by Strub et al. (2003) for spectro-directional terminology. Hess et al. (1976) for taxonomy.

\section{Introduction}

Understanding the relevance to measure biogeophysical parameters using novel techniques arises from various efforts in environmental policy on a global level. The often mentioned Kyoto Protocol to the UN (United Nations) Framework convention on Climate Change (UNFCC) proposes a global policy to be applied at international level, based on assessments of carbon emission and sequestration rates. The aim of the protocol is therefore to stabilize the $\mathrm{CO}_{2}$ concentration in the atmosphere in the end. In particular, the consideration of carbon sinks in the protocol has given a large momentum to implement a scientifically sound accounting and verification system. The key issues to be resolved there are the variability, uncertainty, attribution, non-permanence, leakage, and future evolution of the carbon sequestration in the terrestrial biosphere (Valentini et al. 2000). The estimated carbon uptake of the biosphere must be consistent with all other evidence at three levels of integration of the carbon budget: global, national, and local.

We presently lack the understanding and observations needed to close the annual carbon budget at the global level, though considerable advances are currently being made (Heinsch et al. 2003). Furthermore, it is not yet possible to determine accurately enough the spatial distribu- 
tion and the temporal dynamics of carbon sinks and sources at regional to national scales. This information however, is crucial for policymaking. Recent international research activities highlighted the need for enhanced experimental and monitoring systems (e.g., flux measurements, satellite sensors, field and laboratory experiments, global data archives), which allow for a better parameterisation of terrestrial biosphere models and therefore improve our understanding of the global carbon cycle and its likely future evolution (Nemry et al. 1999).

One particular component of the Earth system, the terrestrial environment has been identified as being a critical component of the variability of the global carbon cycle. However, given the natural diversity of landscapes, the (instrumented) measurement and validation approach remains challenging. In particular, it was found that changing vegetation dynamics play a crucial role in climatevegetation coupling (Cox et al. 2000). Earth observation from airborne or spaceborne platforms is the only observational approach capable of providing data at the relevant scales and resolution needed to extrapolate findings of in situ (field) studies, e.g., the FLUXNET sites (Wilson et al. 2003) to larger areas, to document the heterogeneity of the landscape at regional scale and to connect these findings into a global view. Extrapolation can either be done using geo-statistical methods (Guisan and Zimmermann 2000), as well as by process modelling of ecosystems (Meentemeyer et al. 2001, Schoorl and Veldkamp 2001). The latter is a very promising approach for testing ecological hypotheses and for assessing and forecasting the state of large landscapes up to the global scale. Such approaches usually require the spatial input of the state of the ecosystems at simulation start and of relevant biophysical, biochemical and/or structural information on the terrestrial ecosystems. Ecosystem models - often referred to as biogeochemistry models because they simulate pools and fluxes of relevant ecosystem elements such as carbon, nitrogen or water - ideally combine remote sensing information on the structure of the vegetation with monthly to daily meteorological data and a set of ecophysiological parameters (Thornton et al. 2002), which drive the processes of ecosystems. When applied to a gridded landscape, the combination of spatially explicit air- or spaceborne information on the vegetations structure with ecosystem models allow for an accurate assessment of ecosystem processes, for testing novel ecological theories and for predicting possible future states of the land surface (Kimball et al. 2000, Potter et al. 2001, Turner et al. 2003). Such large scale to global quantifications are clearly beyond the realm of experimental analysis - this contribution therefore focuses on a forest canopy. The close coordination of Earth observation satellites and airborne platforms is thus essential for the successful validation of the contribution of the terrestrial component to the global carbon cycle. Space agencies and international organizations have recently established a coordination mechanism (e.g., the Integrated Global Observing Strategy Partnership (IGOS 2002) that facilitates progress in space-based measurements (Rast et al. 2001).

New Earth observation platform strategies that enable the well calibrated reproducible measurement of the (directional) radiance field of the terrestrial surface at a local, regional (Green et al. 1998), and global scale (Justice et al. 1998) are increasingly becoming operational and available on a larger basis. In particular, various available imaging spectrometers allow closing the scaling gap between local and global for various applications (Myneni et al. 2002, Tian et al. 2003). The derived variables from these measurements are further used to quantify the important (land) processes that are directly relevant for the global carbon cycle. It is demonstrated in this contribution that the technology, science, and applications are available to contribute to the above challenge using a local case study.

\section{Instrumentation approach}

Growing expectations on the use of Earth observation data to support key decisions by governments and industries put increasing pressure on technology to deliver proven, reliable information. Measurements may document small changes in key terrestrial parameters over many years and must finally be related to a global variability in climate change (Nemani et al. 2003). Characterisation of these sensor systems is therefore critical, especially the ones operating in the solar reflected radiation from the Earth's surface (Schaepman and Dangel 2000, Schaepman et al. 2002, Green et al. 2003, Thome et al. 2003).

Imaging spectroscopy today enables the biogeophysical and geochemical variables of the continental biosphere processes to be measured with calibrated physical units and quantifiable accuracy. However, surface characteristics critically govern the involved processes. If we want to take proper actions to mitigate existing uncertainties, surface characteristics must be described accurately to better understand, model and predict the interaction between surface and atmosphere. The complex global models that address these issues use variables and parameters to describe the biophysical and biochemical processes but, when compounded, the uncertainties in these variables and parameters can only produce moderate to poorly accurate results. Amelioration comes from independent information applied to control or constrain the 
models, but the spatially distributed character of land surface processes - large spatial heterogeneity and dynamic character - require remote-sensing observations to provide this information. ESA's (European Space Agency) Living Planet, NASA's (National Aeronautics and Space Administration) EOS programmes and the GMES (Global Monitoring of Environment and Security) initiative recognise this, but there are some remaining unknown error sources due to the inherent uncertainty of the measurement.

\section{The promise of imaging spectroscopy}

Imaging spectroscopy - the quantitative and qualitative characterization of both a surface and the atmosphere using geometrically coherent spectro-radiometric measurements - measures the continuous, upwelling spectral radiance in each spatial pixel (Schaepman et al. 2003a). The results can be used for

- Unambiguous direct and indirect identification of surface materials and atmospheric trace gases,

- Measurement of their relative concentrations,

- Assignment of the proportional contribution of mixed pixel signals (spectral un-mixing problem),

- Derivation of their spatial distribution (mapping problem), and

- Study over time (multi-temporal analysis).

'Conventional multispectral' remote sensing instruments usually cover aspects $4-5$ with sufficient accuracy. New instruments will cover aspects $1-3$, but conventional calibration techniques will still restrict uncertainties to a few percent at best - only marginally adequate for current user demands. Even so, imaging spectroscopy has already supported or been successfully used for Earth system modelling and the corresponding variables have been directly or indirectly quantified using inversion techniques [e.g., snow (Painter et al. 2003); minerals (Kruse et al. 2003); soil (Ben-Dor et al. 2003); water quality (Brando and Dekker 2003); vegetation (Underwood et al. 2003); etc.].

There are a number of existing and planned airborne [e.g., AVIRIS (Airborne Visible Infrared Imaging Spectrometer) and APEX (Airborne Prism Experiment) (Green et al. 1998, Schaepman et al. 2003b)] and spaceborne [e.g., MODIS (Moderate Resolution Imaging Spectrometer), MERIS (Medium Resolution Imaging Spectrometer), MISR (Multiangle Imaging SpectroRadiometer), and SPECTRA (Surface Processes and Ecosystem Changes Through Response Analysis) (Diner et al. 1998, Rast 1999, Rast et al. 2001, Justice and Townshend 2002)] spectro-directional imagers, that will be delivering improved data quality for the further analysis of the directional radiance field.

\section{Relevant vegetation variables and their measurement potential}

The promise of imaging spectroscopy leads to an increased potential to retrieve variables that are relevant in the frame of coupled soil-vegetation-atmosphere systems. The interaction of radiation with canopies and soils depends on the optical, thermal or dielectric properties of the materials as well as on their number, area, orientation and position in space, which constitute the primary biophysical variables. Therefore, remote sensing allows to directly deriving canopy or soil primary biophysical variables. Additionally, secondary variables that are combinations of the primary biophysical variables can also be estimated. The main primary and secondary variables accessible by remote sensing in the solar domain are listed in Table 1.

The information contained in the wavelength range of the solar reflected electromagnetic spectrum (400-2500 $\mathrm{nm})$ provides estimates of canopy structural variables such as LAI and the main biochemical composition: chlorophyll and water content. In addition, Fourty (1997) demonstrated that the canopy integrated biochemical contents (mass of biochemical constituents per unit soil area) were generally more easily accessible than the leaf contents (mass of biochemical constituents per unit leaf area). The canopy-integrated content corresponds to the product of leaf biochemical content and leaf area index. Finally, there is a trend to be observed that future imaging spectrometers will allow the measurement of pigments (Sims and Gamon 2002) and fluorescence (Zarco-Tejada et al. 2003) of vegetation, contributing to the above list of variables.

By summarizing the key variables that can be derived using imaging spectrometer-based Earth observation techniques, Table 2 can be compiled. A listing of variables (left) is compared with their retrieval uncertainty using 'conventional' satellite systems with two spectral bands and one view angle (middle), and current highresolution spectro-directional sensors based on a radiative transfer modelling exercise.

\section{Linking local to global scales}

Most of the existing field measurements and sampling schemes take place at a local scale between a few and hundred meters, corresponding to a typical length scale of the 
Table 1. Retrieval potential of biophysical variables as a function of two generalised spectral domains. The accuracy requirements are listed as input requirements into models (Baret 2001). The level of accuracy and robustness of the estimation is indicated by the symbol "+" (“++++" accurate and robust; "-“ no estimates possible).

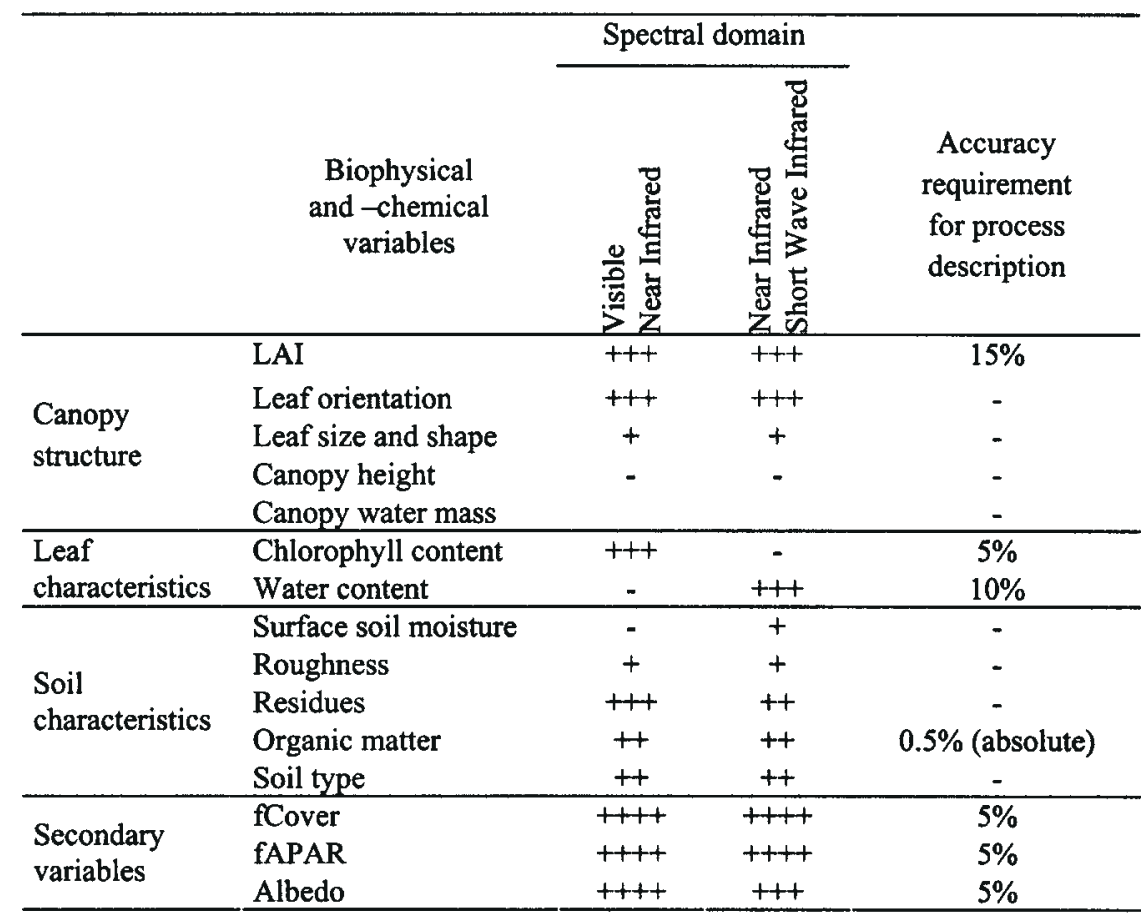

Table 2. Modelled retrieval error for key properties of terrestrial vegetation, based on results of direct and inverse radiative transfer modelling (Rast et al. 2001). 'Not feasible' indicates that the retrieval is not feasible with sufficient accuracy to be useful.

\begin{tabular}{lcc}
\hline Variables & \multicolumn{2}{c}{ Sampling of angular and spectral dimensions } \\
\cline { 2 - 3 } & 1 view angle, 2 spectral bands & Multiangular, hyperspectral \\
\hline fCover & $40 \%$ & $5 \%$ \\
fAPAR & $30 \%$ & $5 \%$ \\
Albedo & $30 \%$ & $2 \%$ \\
LAI & $125 \%$ & $10 \%$ \\
Leaf chlorophyll & $85 \%$ & $10 \%$ \\
Leaf water & Not feasible & $20 \%$ \\
Leaf dry matter & Not feasible & $20 \%$ \\
Foliage temperature & Not feasible & $1^{\circ} \mathrm{K}$ \\
Soil temperature & Feasible & $2^{\circ} \mathrm{K}$ \\
Fraction living/dead & Not feasible & $20 \%$ \\
biomass & & \\
\hline
\end{tabular}

internal variability of landscapes [such as the global spatially distributed FLUXNET towers (Wilson et al. 2003)]. Current practise of scaling from local to global based on local field measurements can result in significantly varying uncertainties and errors. This is because vegetation is heterogeneous at a wide range of spatial scales. Significant efforts are therefore being made in spaceborne instrumentation to measure biome (or ecosystem) specific heterogeneity of variables and processes at a regional scale (up to $100 \mathrm{~km}$ ), while maintaining the local scale explic- itly. This is achieved by regional case studies, considering the spatial and temporal gradients of the biome under investigation. The proposed SPECTRA mission (Rast et al. 2001) will help developing robust, detailed Earth System Models over a representative ensemble of biomes and conditions. In the following case study, we demonstrate how imaging spectroscopy can be useful to quantitatively retrieve biogeophysical characteristics from a subalpine forest representative for boreal forests. 


\section{Case study: estimation of canopy parameters within a mountain forest}

The study area for the acquisition of the field data is located in the Eastern Ofenpass valley $\left(10^{\circ} 13^{\prime} 48^{\prime \prime} \mathrm{E} /\right.$ $46^{\circ} 39^{\prime} 45^{\prime \prime} \mathrm{N}$ ), which is part of the Swiss National Park (SNP), Switzerland. The Ofenpass represents an inner-alpine valley on an average altitude of about $1900 \mathrm{~m}$ a.s.1 with annual precipitation of 900-1100 mm. Embedded in this environment are boreal type subalpine forests. The south-facing Ofenpass forests, the location of the field measurements, are largely dominated by mountain pine (Pinus montana ssp. arborea) and some stone pine (Pinus cembra) as a second tree species. These forest stands can be classified as woodland associations of the EricoPinetum mugo. The understory is characterized by low and dense vegetation composed mainly of various Ericaceae and Sesleria species.

\section{Field sampling for radiative transfer modelling}

Four core test sites (labelled LWF1 and 2, STA1 and 2) and several additional distributed point samples are characterized to describe the canopy and the spectral characteristics of the study area. The core test sites are selected following a stratified sampling scheme to cover different canopy densities within a stand of Pinus montana ssp. arborea (Fig.1).
The choice of test sites is defined following the elementary sampling units of the VALERI scheme (INRA 2003, Jonckheere et al. 2004, Weiss et al. 2004, Baret et al. in press). Each site is defined by nine sampling points, evenly spaced in a grid of 10 meters, covering an area of $20 \mathrm{~m} \times 20 \mathrm{~m}$. The coordinates of the sampling points are georeferenced by GPS (Global Positioning System) receivers. Measurements of the biophysical and biochemical variables describing the canopy are performed at all test sites dated from August 7-15, 2002. Mean values of these measurements are presented in Table 3 .

The canopy structure is measured using two canopy analyzers LAI2000 and hemispherical photographs providing canopy structure variables separately for the crown and understory layer. The clumping effects at the shoot and crown level, typical for coniferous foliage, are corrected following an approach proposed by Chen et al. (1997). Observed LAI values ranged between 1.78 and 3.99 , whereas the measurement uncertainties amounted to $22 \%$. Hemispherical photographs taken parallel to the LAI2000 measurements allowed the separation of the canopy into its constituent foliage and wood fractions, i.e., needles, trunk and branches. Forest stand characteristics such as stem density, tree height and crown radius were measured over an area of two hectares containing a total of 2456 trees. Additionally, the height of the crown

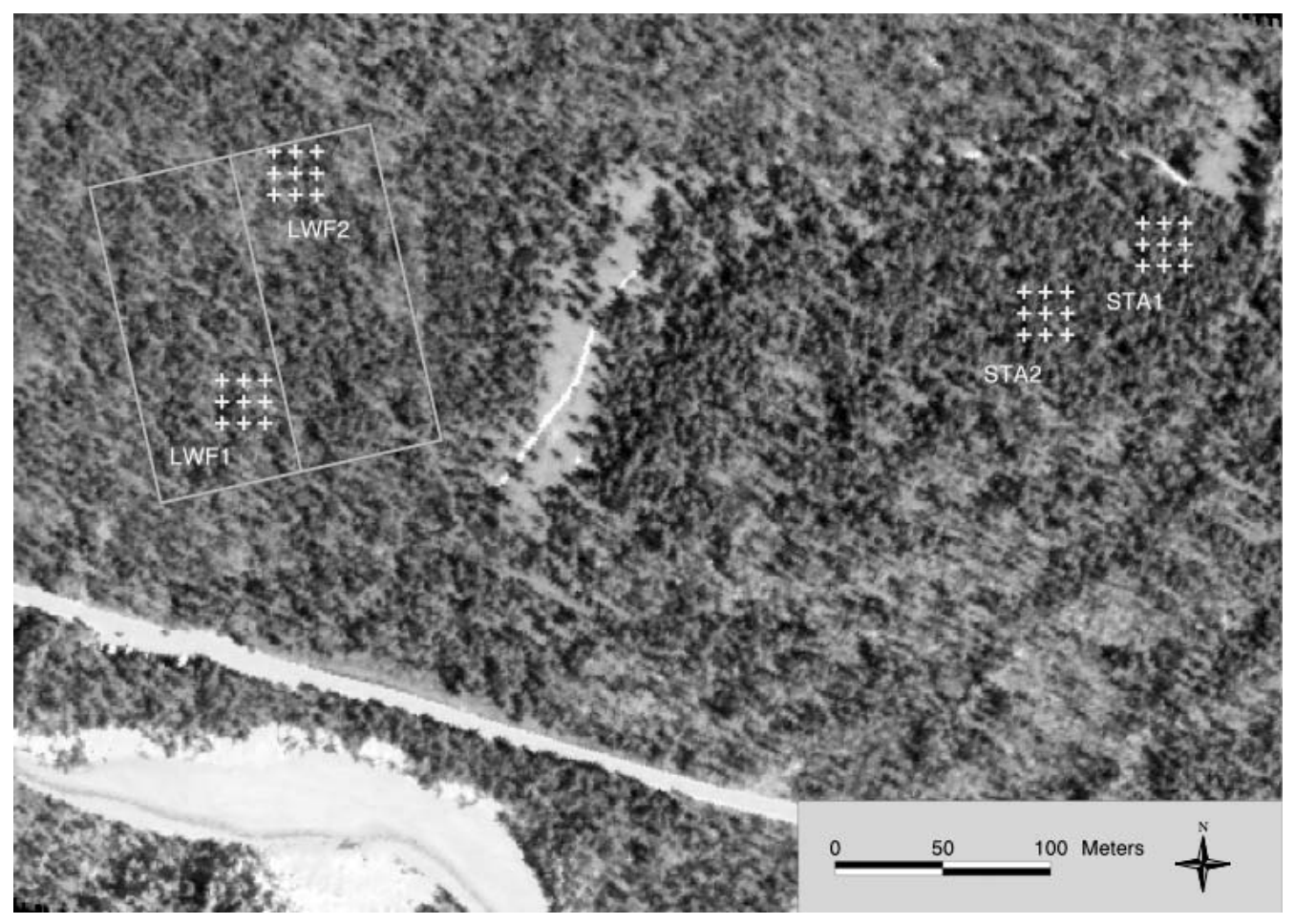

Figure 1. Airborne imaging spectrometer data acquired over the four core test sites (indicated by crosses) as part of the Ofenpass test site within the Swiss National Park (SNP). The four test sites are $20 \mathrm{~m}$ x $20 \mathrm{~m}$ in spatial extent and have been sampled with the variables listed in Table 3. 
Table 3. Field observations of canopy variables including relative measurement errors (denoted in brackets) relevant for the canopy parameterization as an input to the radiative transfer models (RTM). The spectral properties of the woody parts and understory are characterized by spectroradiometric field measurements. There are 9 measurement points per unit (U) listed and for each measurement point $n$ measurements have been taken. Structural parameters are all averaged from $n=36$ measurements.

\begin{tabular}{|c|c|c|c|c|c|}
\hline Parameter & Units & LWF1 & LWF2 & STA1 & STA2 \\
\hline \multicolumn{6}{|l|}{ Foliage } \\
\hline $\begin{array}{l}\text { Water content }(\mathrm{U}=4 \text {, } \\
\mathrm{n}=1)\end{array}$ & $\mathrm{g} / \mathrm{cm}^{2}$ & $\begin{array}{c}0.047 \\
(7.5 \%)\end{array}$ & $\begin{array}{c}0.045 \\
(7.5 \%)\end{array}$ & $\begin{array}{c}0.049 \\
(7.5 \%)\end{array}$ & $\begin{array}{c}0.042 \\
(7.5 \%)\end{array}$ \\
\hline Dry matter $(U=4, n=1)$ & $\mathrm{g} / \mathrm{cm}^{2}$ & $\begin{array}{l}0.038 \\
(7.5 \%)\end{array}$ & $\begin{array}{c}0.036 \\
(7.5 \%)\end{array}$ & $\begin{array}{c}0.038 \\
(7.5 \%)\end{array}$ & $\begin{array}{l}0.035 \\
(7.5 \%)\end{array}$ \\
\hline $\begin{array}{l}\text { Chlorophyll content } \\
(U=4)\end{array}$ & $\mu \mathrm{g} / \mathrm{cm}^{2}$ & $\begin{array}{c}61.8 \\
(1.54 \%)\end{array}$ & $\begin{array}{c}75.1 \\
(1.54 \%)\end{array}$ & $\begin{array}{c}59.0 \\
(1.54 \%)\end{array}$ & $\begin{array}{c}62.8 \\
(1.54 \%)\end{array}$ \\
\hline $\begin{array}{l}\text { Mesophyll structure ( } \\
\mathrm{n}=7 \text { ) }\end{array}$ & Unitless & \multicolumn{4}{|c|}{$3.78(22 \%)$} \\
\hline \multicolumn{6}{|c|}{ Canopy structure (overstory) } \\
\hline LAI $(U=4, n=5)$ & Unitless & $2.18(13 \%)$ & $1.78(22 \%)$ & $3.89(19 \%)$ & $3.99(17 \%)$ \\
\hline $\begin{array}{l}\text { Fractional cover }(U=4, \\
n=5)\end{array}$ & $\%$ & $55 \%(13 \%)$ & $46 \%(22 \%)$ & $77 \%(19 \%)$ & $79 \%(17 \%)$ \\
\hline $\begin{array}{l}\text { Wood fraction }(\mathrm{U}=4 \text {, } \\
\mathrm{n}=1)\end{array}$ & $\%$ & $30 \%$ & $30 \%$ & $30 \%$ & $40 \%$ \\
\hline Crown shape & n.a. & \multicolumn{4}{|c|}{ Cone } \\
\hline Tree distribution & n.a. & \multicolumn{4}{|c|}{ Poisson distribution } \\
\hline Tree height & $\mathbf{m}$ & \multicolumn{4}{|c|}{$11.93 \pm 2.9$} \\
\hline Crown radius & $\mathrm{m}$ & \multicolumn{4}{|c|}{1.765} \\
\hline Crown base & $\mathrm{m}$ & \multicolumn{4}{|c|}{7} \\
\hline Trunk diameter & $\mathrm{m}$ & \multicolumn{4}{|c|}{0.179 (at ground) } \\
\hline $\begin{array}{l}\text { Crown height width } \\
\text { ratio }\end{array}$ & Unitless & \multicolumn{4}{|c|}{2.83} \\
\hline Hotspot & Unitless & \multirow{2}{*}{\multicolumn{4}{|c|}{$\begin{array}{c}0.1 \\
\text { Average leaf angle: } 58.43^{\circ} \text { (foliage), } 30^{\circ} \text { (woody parts) }\end{array}$}} \\
\hline Leaf angle distribution & $\operatorname{deg}$ & & & & \\
\hline
\end{tabular}

base was visually estimated during the field campaign of this study.

Standard wet-laboratory procedures are used for determination of foliage water, chlorophyll content and dry matter. The samples are collected from the upper part of the tree crowns, each consisting of one branch carrying newly developed and old needles. The difference between fresh and dry weight allowed for the calculation of water content expressed either as relative value per unit mass (Fuel Moisture Content, FMC \%) or per unit leaf area as Equivalent Water Thickness (EWT expressed in either $\left[\mathrm{g} / \mathrm{cm}^{2}\right]$ or $\left.[\mathrm{cm}]\right)$. The concentration of photosynthetic pigments (Chl. $a$ and $b$ ) within the foliage is determined using a CADAS 100 spectrophotometer. Following the equations of (Lichtenthaler 1987) the pigment concentrations were converted to $\left[\mu \mathrm{g} / \mathrm{cm}^{2}\right]$. The observed biochemical concentrations showed only a low variability (Table 3). The mesophyll structure parameter $\mathrm{N}$ was inverted by iterative minimization of PROSPECT from the average foliage reflectance measured with the Licor1800 integrating sphere, while the biochemistry was set to stand values. The uncertainty of the $\mathrm{N}$ parameter estimation was assessed by inversion over the variability of fo- liage reflectance measurements. The foliage reflectance showed a high variability due to errors in the assessment of gaps within the observed foliage sample. Consequently, the $\mathrm{N}$ parameter was subject to an uncertainty of $22 \%$.

The spectral properties of several canopy components such as the reflectance of the understory, woody parts and the foliage have been measured using a field spectrometer (ASD FieldSpec Pro FR). Field spectra are collected during the over flight $1.5 \mathrm{~m}$ above the ground and within 2 hours of solar noon under clear sky conditions (Figure 2). In addition, the spectral characteristics of branches and bark of trunks are assessed from several samples. For the understory, reflectance transects consisting of 10 to 30 spectroradiometric measurements were acquired at each test site. The acquisition of the reflectance of coniferous foliage involved an ASD field spectroradiometer coupled with an integrating sphere (LiCor 1800) and a custommade light source for improved illumination. The gap fraction of samples not covering the instrument port was assessed with a high-resolution digital camera and subsequent image analysis. The gap effects on the reflectance 
measurements were corrected by taking proportionally into account the spectral properties of the background.

The imaging spectrometer data were acquired on $\mathrm{Au}-$ gust 14, 2002 simultaneously with the DAIS 7915 (Digital Airborne Imaging Spectrometer) and ROSIS (Reflective Optics Imaging Spectrometer) imaging spectrometers parallel to the ground measurements. The spectrometers are operated onboard of the DLR (German Aerospace Centre) Dornier DO-228 aircraft. The local illumination and observation conditions were a solar zenith angle of $45.3^{\circ}$, a solar azimuth angle of $122.9^{\circ}$, and the flight heading of $293^{\circ}$. There was no cloud coverage at the time of the overflight. This study concentrates on the data recorded by the DAIS7915 imaging spectrometer, covering the spectral range from the visible to the thermal infrared (VIS/NIR: 0.5-1.1 $\mu \mathrm{m}$, SWIR1: 1.6-1.8 $\mu \mathrm{m}$, SWIR2: 2-2.5 $\mu \mathrm{m}$, MIR: 3-5 $\mu \mathrm{m}$, TIR: 8.7-13 $\mu \mathrm{m}$ ) with 79 bands. The airborne campaign was organized to cover the Ofenpass valley, providing imaging spectrometer data in a spatial resolution of five meters. The flight line was oriented close to the solar principal plane sun during the overflight time to minimize directional effects. The images were geo-atmospherically processed with the modules PARGE and ATCOR4 to obtain geocoded top-ofcanopy reflectances (Richter and Schlapfer 2002, Schlapfer and Richter 2002). Concurrent spectroradiometric measurements of selected reference targets in the field also allowed a validation of the retrieved surface reflectance and a subsequent vicarious calibration of the imaging spectrometer data (Secker et al. 2001). The quality of the vicarious calibration and radiometric correction was assessed taking ground spectroradiometric measure- ments of a homogeneous meadow as reference. The reflectance derived from the imaging spectrometer DAIS7915 yielded absolute differences relative to ground reflectance of $0.4 \%$ in the VIS and $0.8 \%$ in the NIR, which corresponds to $8 \%$ (VIS) respectively $2 \%$ (NIR) of relative deviation. An image quality assessment at radiance level revealed a list of bad bands, which were discarded from analysis, thus leaving 34 bands in the wavelength range of $0.5-1.8 \mu \mathrm{m}$.

\section{Radiative transfer modelling for canopy parameter estimation}

The hybrid radiative transfer model (RTM) called the GeoSAIL (Huemmrich 2001) is used to describe canopy reflectance at scene level. A scene has been defined in this study as being an area of $30 \mathrm{~m} \times 30 \mathrm{~m}$ with the requirement that crown or shadow fractions are small compared to the total area modelled. The radiative transfer at foliage level is characterized by the PROSPECT model (Jacquemoud et al. 1996), which provides the foliage optical properties as a function of the biochemistry and is then subsequently coupled with the canopy RTM. The RTM GeoSAIL describes the canopy reflectance of a complete scene including discontinuities in the canopy and shadowed scene components. The RTM combines a geometric model with the SAIL model (Verhoef 1984) which provides the reflectance and transmittance of the tree crowns. The geometric model determines the fraction of the illuminated and shadowed scene components as a function of canopy coverage, crown shape and illumination angle. All trees are assumed to be identical with no crown overlap nor does the model account for mutual shading. The
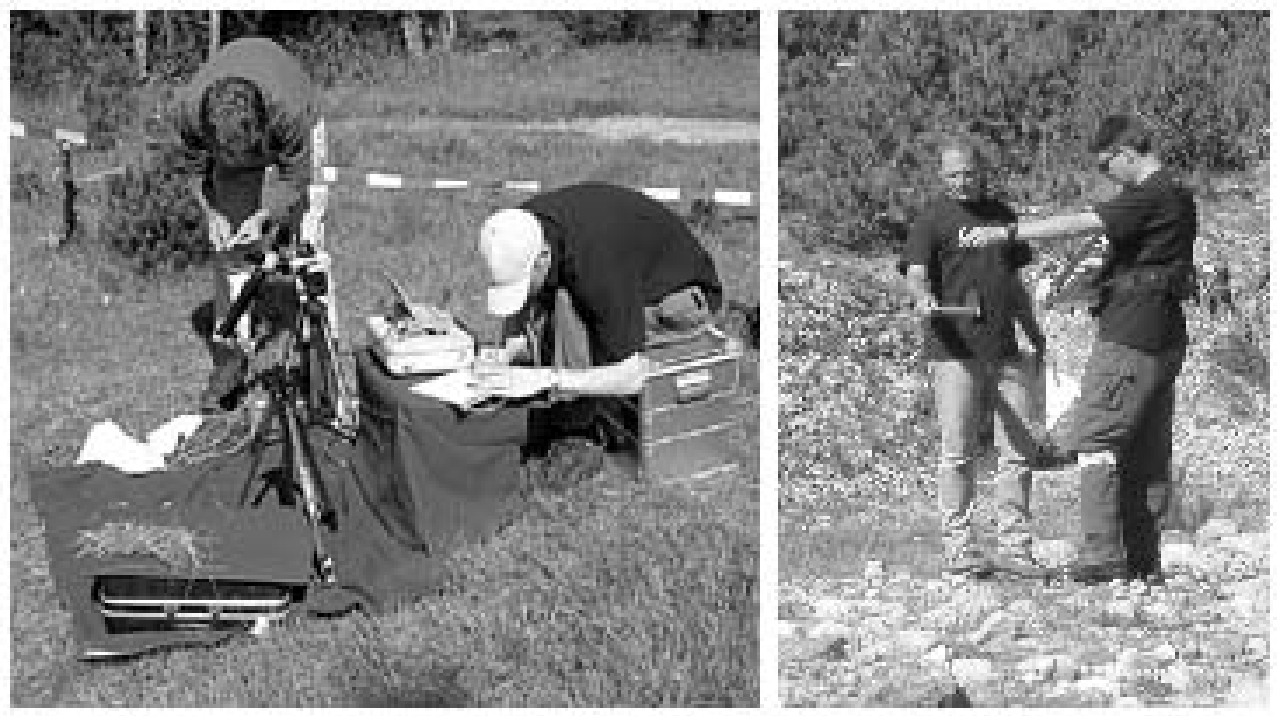

Figure 2. Spectroradiometric measurements of needle optical properties during the imaging spectrometer overflight: needle reflectance and transmittance (left), and ground reflectance (right). 
Table 4. Specific ranges for each parameter describing the constraining space for canopy realizations. These boundaries are used for the generation of the look up table.

\begin{tabular}{lccc}
\hline \multicolumn{1}{c}{ RTM parameter } & Unit & Minimum & Maximum \\
\hline LAI & unitless & 1 & 5 \\
Fractional cover & $\%$ & 0.4 & 0.85 \\
Wood fraction & $\%$ & 0.25 & 0.45 \\
Chlorophyll content & $\mu \mathrm{g} / \mathrm{cm}^{2}$ & 55 & 80 \\
Water content & $\mathrm{g} / \mathrm{cm}^{2}$ & 0.025 & 0.065 \\
Dry matter & $\mathrm{g} / \mathrm{cm}^{2}$ & 0.02 & 0.05 \\
$\mathrm{~N}$ & unitless & 2 & 5 \\
\hline
\end{tabular}

radiative transfer within the crowns is calculated using SAIL, which considers the canopy as a horizontal, homogeneous, turbid and infinitely extended vegetation layer composed of lambertian scatterers. The SAIL version within GeoSAIL is adapted to account for the contribution of multiple canopy components with different optical properties, leaf area index and foliage inclination angles, but is limited to ten wavelength bands. For the coupling of GeoSAIL with PROSPECT a SAIL version (Weiss et al. 2001) capable of dealing with an unlimited number of bands and multiple canopy components, such as foliage and branches, was used.

The radiative transfer is parametrized at the foliage and canopy level by the input parameters of PROSPECT and GeoSAIL, describing the biochemical and biophysical properties of the canopy (Table 3). (For the inversion the field measurements are only relevant for the LUT range definition - see below. But some parameters had to be fixed for the inversion, which is why they are described below and in Table 3). The average inclination angle could be parameterized separately for the two foliage elements in GeoSAIL and were set to a spherical distribution for green foliage and plagiophile distribution for woody parts. The tree geometry relevant to GeoSAIL was based on the forest stand characteristics describing tree height, crown radius and crown length. The trees were horizontally distributed within the scene according to the Poisson distribution. The spectral properties of the remaining canopy components such as the understory and woody parts were characterized by ground spectroradiometric measurements and were assumed to be inherent to all test sites. Woody parts were treated as opaque foliage elements, thus only reflecting or absorbing incident radiation. The structural parameterization within the crown relied on the total LAI of the overstory, corrected for clumping effects. The derived wood fraction allowed resolving the total overstory LAI into its green foliage and woody parts.

Inversion of GeoSAIL

The inversion of GeoSAIL was based on look up tables (LUT), whose generation consisted in precomputing the canopy reflectance for 130000 canopy realizations and considering the measurement configuration. The parameters corresponding to each canopy realization were randomly drawn following a uniform distribution. The range of each variable was defined based on ground measurements performed in this study and on experimental data presented in the literature (Dungan et al. 1996, Chen et al. 1997, Gond et al. 1999, Ceccato et al. 2001) (Table 4). The selected ranges corresponded to a distribution of the respective variable typical for the observed coniferous canopy. The generation of the LUT allowed consequently for the implementation of general prior information depending on the specific vegetation type. Tree geometry and spectral properties of the understory and woody parts were also specified by the forest stand characteristics and ground measurements.

The model inversion was carried out by minimizing the merit function $\chi^{2}$, as depicted in Eq. 1, and is defined as the distance between the canopy reflectance $\rho_{\text {mes }}$ acquired by the DAIS 7915 and the simulated reflectance $\rho_{\text {sim }}$ found in the LUT. The distance criterion was weighted using the uncertainty of the spectroradiometric measurements. $\delta_{\text {DAIS }}$ is related to calibration of the DAIS 7915 sensor and the atmospheric correction of the imaging spectrometer data:

$$
\chi^{2}=\sum_{i=1}^{n_{\lambda}} \frac{1}{\delta_{D A I S}}\left(\rho_{m e s}^{i}-\rho_{\text {sim }}^{i}\right)^{2},
$$

where $n_{\lambda}$ is the number of finally included imaging spectrometer bands (e.g., 39). Canopy realizations found within a tolerance of $20 \%$ of the minimal calculated distance $\chi^{2}$ were considered as possible solutions; their median defined the final solution and their standard deviation the uncertainty of the inversion.

\section{Results}

We are using forward simulation of canopy reflectance and an inversion of a radiative transfer models to estimating forest canopy variables. Inverting GeoSAIL 
for measured canopy reflectance subsequently assessed the feasibility of estimating canopy variables employing a radiative transfer model. Figure 3 presents the final results of the forest variable estimation relative to the ground measurements of the respective variable, while also indicating the uncertainties associated to the inversion process and ground measurements. The model inversion performed well with reasonable root mean square errors (RMSE; c.f., Figure 3) and within uncertainties for the variables describing the canopy structure, such as LAI and fractional cover. The estimation of the foliage variables provided less stable results, but the average accu- racy of estimates still amounted to $71.6 \%$ and $78.2 \%$, respectively for foliage water content and dry matter. A limitation was due to the variation of foliage variables, which was not sufficiently large for a thorough validation due to the observed canopy homogenous in terms of species, phenology and environmental conditions. The estimation of chlorophyll foliage content showed poor results probably due to the effects already observed and discussed for the simulated canopy reflectance. The product of LAI and foliage water or dry matter content represented the canopy content of the respective biochemical constituent, which could present a quantity most relevant to
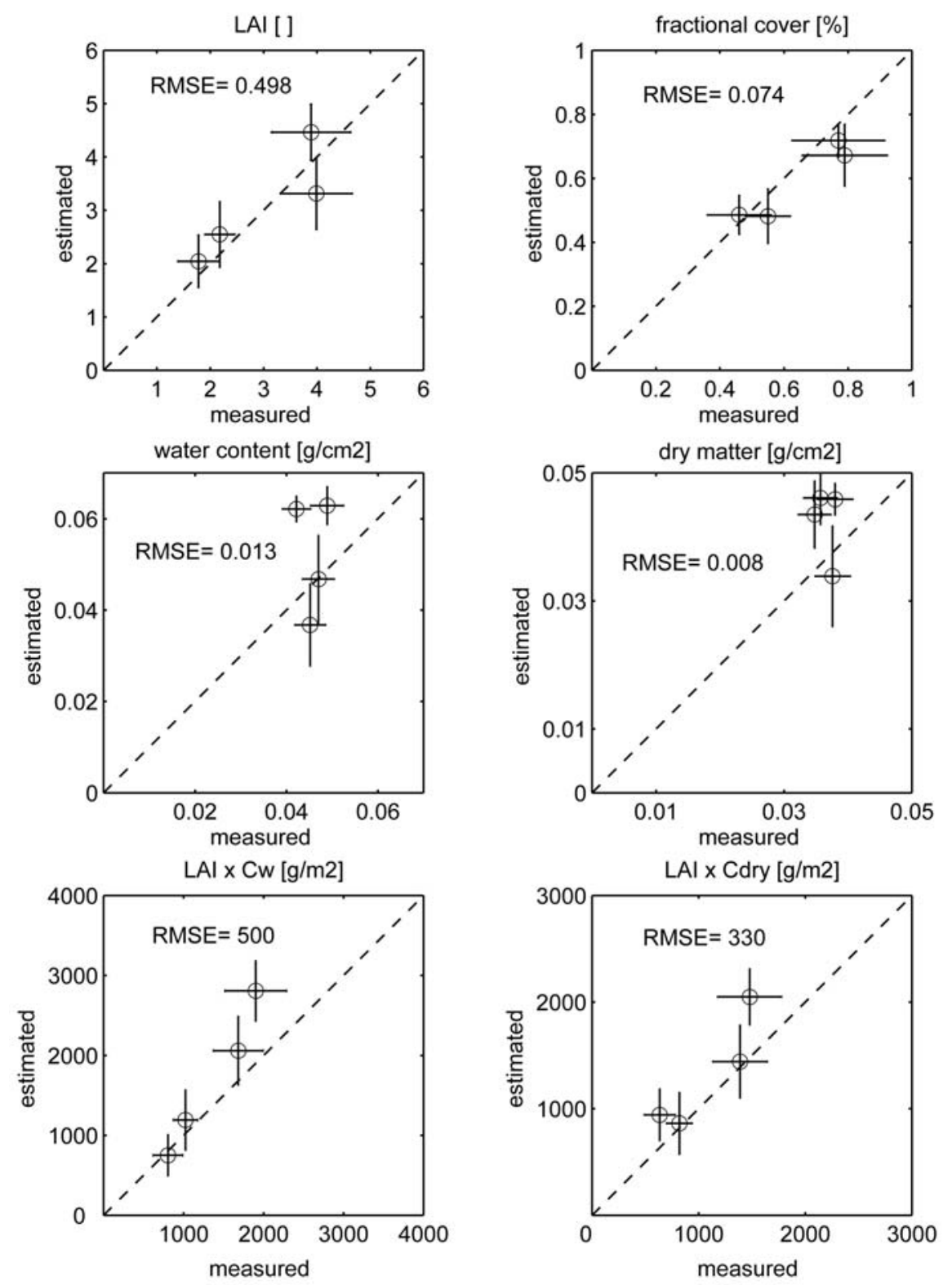

Figure 3. Performance of radiative transfer model inversion: estimates and measurements of canopy parameters related to the four test sites. Error bars represent the uncertainties related to the ground measurements (e.g., the nine plots summarised into the four test sites) and model inversion respectively. LAI x Cw: canopy water content, LAI x Cdry: Canopy dry matter content, RMSE: root mean square error in corresponding units. 
the inflammability and the combustion of forests (Ceccato et al. 2002).

\section{Conclusions}

Imaging spectroscopy - even though being a relatively new area in Earth remote sensing - has demonstrated over the past few years its unique potential to retrieve quantitatively biogeophysical and biogeochemical parameters. The particular success of imaging spectroscopy can be attributed to the improved data quality (more mature technology, calibration stability, increased number of spectral bands and observation angles, high signal-to-noise-ratio), wider availability of consistent observations to the user community, and emerging new applications. Imaging spectrometers will continue to help advance remote sensing science and technology by:

- Assisting to design new or better quantitative based algorithms for the exploitation of current and future sensors,

- Defining more appropriate observational requirements for Earth observation related sensors that will be needed in the coming years, and

- Reanalyzing measurements obtained in the past with less sophisticated sensors.

Nevertheless, long-term observations or trends detected and analyzed using these imaging spectrometers are still a few years to go due to the inherent short living period of these instruments.

By using a regionally based case study in a mountain forest, we demonstrate the successful canopy variable estimation in a heterogeneous canopy. The coupled model approach using PROSPECT and GeoSAIL allow a quantitative approach for the extraction of relevant vegetation variables. The canopy variables provide information on the vegetation status vital to the management of forests with respect to ecological modelling. The hyperspectral extension of GeoSAIL supports also the robust and reliable retrieval of a combined assessment of biophysical and biochemical variables. Intensive ground validation for radiative transfer based approaches supported the generation of a look up table with site-specific model parameter constraints. The derived canopy variables represent the actual spatial distribution of forest biophysical and biochemical properties as they occur in the landscape.

Improvements to the proposed approach may include the use of more sophisticated radiative transfer models (e.g., FLIGHT), which represent in a more sophisticated manner the link of physical properties between the remote sensing system and the ecosystem observed. On the struc- tural parameter side, the geometrical representation of the canopy derived from a LIDAR system would offer an optimal complement to the radiometric information (Koetz et al. 2003).

The increased spatial resolution of quantitative information of forest conditions provides a sound data basis relevant to forest management unities and to the quantification of ecosystem pools (e.g., carbon). The fine spatially distributed information available on canopy variables also allows defining realistic initial starting and boundary conditions of ecological models on a spatial scale improving their representation of ecological processes. Finally, such data are very valuable for testing ecological hypotheses and model predictions, due to their spatially explicit character and high spectral resolution.

Acknowledgements. Parts of this contribution are funded by the EC project 'Forest Fire Spread and Mitigation (SPREAD) (EC-Contract Nr. EVG1-CT-2001-00027 through the Federal Office for Education and Science of Switzerland (BBW), (BBW-Contract Nr. 01.0138)). Ground measurements and acquisition were supported by RSL staff, WSL (tree sampling), INRA (leaf optical measurement equipment). The airborne operations have been carried out in the framework of the EU Access to Infrastructure project HYSENS, under guidance of DLR. The access permission and support of field logistics has been given by the SNP through Britta Allgöwer. We would like to thank the anonymous reviewers for helpful suggestions and comments.

\section{References}

Baret, F. 2001. Variables associated to vegetation processes and retrieval prospects from high spectral resolution imaging systems. In: M. Schaepman (ed.), Performance and Calibration Requirements for APEX. Remote Sensing Laboratories, Zurich, pp. 86101.

Baret, F., M. Weiss, S. Garrigue, D. Allard, J. P. Guinot, M. Leroy, H. Jeanjean, H. Bohbot, R. Bosseno, G. Dedieu, C. Di Bella, M. Espana, V. Gond, X. F. Gu, D. Guyon, C. Lelong, E. Mougin, T. Nilson, F. Veroustraete and R. Vintilla. in press. VALERI: a network of sites and a methodology for the validation of medium spatial resolution land satellite products. Remote Sensing of Environment.

Ben-Dor, E., N. Goldlshleger, Y. Benyamini, M. Agassi and D. G. Blumberg. 2003. The spectral reflectance properties of soil structural crusts in the 1.2- to $2.5-\mu \mathrm{u} \mathrm{m}$ spectral region. Soil Science Society of America Journal 67:289-299.

Brando, V. E. and A. G. Dekker. 2003. Satellite hyperspectral remote sensing for estimating estuarine and coastal water quality. IEEE Transactions on Geoscience and Remote Sensing 41:13781387.

Ceccato, P., S. Flasse and J. M. Gregoire. 2002. Designing a spectral index to estimate vegetation water content from remote sensing data - Part 2. Validation and applications. Remote Sensing of Environment 82:198-207.

Ceccato, P., S. Flasse, S. Tarantola, S. Jacquemoud and J. M. Gregoire. 2001. Detecting vegetation leaf water content using reflectance in the optical domain. Remote Sensing of Environment 77:22-33. 
Chen, J. M., P. M. Rich, S. T. Gower, J. M. Norman and S. Plummer. 1997. Leaf area index of boreal forests: Theory, techniques, and measurements. Journal of Geophysical Research-Atmospheres 102:29429-29443.

Cox, P. M., R. A. Betts, C. D. Jones, S. A. Spall and I. J. Totterdell. 2000. Acceleration of global warming due to carbon-cycle feedbacks in a coupled climate model. Nature 408:184-187.

Diner, D. J., J. C. Beckert, T. H. Reilly, C. J. Bruegge, J. E. Conel, R. A. Kahn, J. V. Martonchik, T. P. Ackerman, R. Davies, S. A. W. Gerstl, H. R. Gordon, J. P. Muller, R. B. Myneni, P. J. Sellers, B. Pinty and M. M. Verstraete. 1998. Multi-angle Imaging SpectroRadiometer (MISR) - Instrument description and experiment overview. IEEE Transactions on Geoscience and Remote Sensing 36:1072-1087.

Dungan, J., L. Johnson, C. Billow, P. Matson, J. Mazzurco, J. Moen and V. Vanderbilt. 1996. High spectral resolution reflectance of douglas fir grown under different fertilization treatments: Experiment design and treatment effects. Remote Sensing of Environment 55:217-228.

Gond, V., D. G. G. de Pury, F. Veroustraete and R. Ceulemans. 1999. Seasonal variations in leaf area index, leaf chlorophyll, and water content; scaling-up to estimate fAPAR and carbon balance in a multilayer, multispecies temperate forest. Tree Physiology 19:673-679.

Green, R. O., M. L. Eastwood, C. M. Sarture, T. G. Chrien, M. Aronsson, B. J. Chippendale, J. A. Faust, B. E. Pavri, C. J. Chovit, M. S. Solis, M. R. Olah and O. Williams. 1998. Imaging spectroscopy and the airborne visible infrared imaging spectrometer (AVIRIS). Remote Sensing of Environment 65:227248.

Green, R. O., B. E. Pavri and T. G. Chrien. 2003. On-orbit radiometric and spectral calibration characteristics of EO-1 Hyperion derived with an underflight of AVIRIS and in situ measurements at Salar de Arizaro, Argentina. Ieee Transactions on Geoscience and Remote Sensing 41:1194-1203.

Guisan, A. and N. E. Zimmermann. 2000. Predictive habitat distribution models in ecology. Ecological Modelling 135:147-186.

Heinsch, F. A., M. Reeves, C. F. Bowker, P. Votava, S. Kang, C. Milesi, M. Zhao, J. Glassy, W. M. Jolly, J. S. Kimball, R. R. Nemani and S. W. Running. 2003. User's Guide: GPP and NPP (MOD17A2/A3) Products NASA MODIS Land Algorithm. Missoula, MT, USA.

Hess, H. H., E. Landolt and R. Hirzel. 1976. Flora der Schweiz und angrenzender Gebiete, 2nd edition. Birkhauser, Basel.

Huemmrich, K. F. 2001. The GeoSail model: a simple addition to the SAIL model to describe discontinuous canopy reflectance. Remote Sensing of Environment 75:423-431.

IGOS. 2002. Integrated Global Observing Strategy http://ceos.esa.int/igosp9/ in ESA.

INRA. 2003. Validation of Land European Remote Sensing Instruments (VALERI). In http://www.avignon.inra.fr/valeri. INRA, Avignon.

Jacquemoud, S., S. L. Ustin, J. Verdebout, G. Schmuck, G. Andreoli and B. Hosgood. 1996. Estimating leaf biochemistry using the PROSPECT leaf optical properties model. Remote Sensing of Environment 56:194-202.

Jonckheere, I., S. Fleck, K. Nackaerts, B. Muys, P. Coppin, M. Weiss and F. Baret. 2004. Review of methods for in situ leaf area index determination - Part I. Theories, sensors and hemispherical photography. Agricultural and Forest Meteorology 121:19-35.

Justice, C. and J. Townshend. 2002. Special issue on the moderate resolution imaging spectroradiometer (MODIS): a new genera- tion of land surface monitoring. Remote Sensing of Environment $83: 1-2$.

Justice, C. O., E. Vermote, J. R. G. Townshend, R. Defries, D. P. Roy, D. K. Hall, V. V. Salomonson, J. L. Privette, G. Riggs, A. Strahler, W. Lucht, R. B. Myneni, Y. Knyazikhin, S. W. Running, R. R. Nemani, Z. M. Wan, A. R. Huete, W. van Leeuwen, R. E. Wolfe, L. Giglio, J. P. Muller, P. Lewis and M. J. Barnsley. 1998. The Moderate Resolution Imaging Spectroradiometer (MODIS): Land remote sensing for global change research. IEEE Transactions on Geoscience and Remote Sensing 36:1228-1249.

Kimball, J. S., A. R. Keyser, S. W. Running and S. S. Saatchi. 2000. Regional assessment of boreal forest productivity using an ecological process model and remote sensing parameter maps. Tree Physiology 20:761-775.

Koetz, B., F. Morsdorf, M. Schaepman, E. Meier, K. Itten and B. Allgöwer. 2003. Specific Parameterization of a Boeral Forest for a 3-D Radiative Transfer Modelling Approach based on Imaging Spectrometer Data and High Resolution LIDAR Data. In Proc. ESA-SSP on SPECTRA, Noordwijk.

Kruse, F. A., J. W. Boardman and J. F. Huntington. 2003. Comparison of airborne hyperspectral data and EO-1 Hyperion for mineral mapping. Ieee Transactions on Geoscience and Remote Sensing 41:1388-1400.

Lichtenthaler, H. K. 1987. Chlorophylls and cartenoids: pigments of photosynthetics biomembranes. Methods of Enzymology 148:350-382.

Martonchik, J. V., C. J. Bruegge and A. Strahler. 2000. A review of reflectance nomenclature used in remote sensing. Remote Sensing Reviews 19:9-20.

Meentemeyer, R. K., A. Moody and J. Franklin. 2001. Landscapescale patterns of shrub-species abundance in California chaparral - The role of topographically mediated resource gradients. Plant Ecology 156:19-41.

Myneni, R. B., S. Hoffman, Y. Knyazikhin, J. L. Privette, J. Glassy, Y. Tian, Y. Wang, X. Song, Y. Zhang, G. R. Smith, A. Lotsch, M. Friedl, J. T. Morisette, P. Votava, R. R. Nemani and S. W. Running. 2002. Global products of vegetation leaf area and fraction absorbed PAR from year one of MODIS data. Remote Sensing of Environment 83:214-231.

Nemani, R. R., C. D. Keeling, H. Hashimoto, W. M. Jolly, S. C. Piper, C. J. Tucker, R. B. Myneni and S. W. Running. 2003. Climate-driven increases in global terrestrial net primary production from 1982 to 1999 . Science 300:1560-1563.

Nemry, B., L. Francois, J. C. Gerard, A. Bondeau and M. Heimann. 1999. Comparing global models of terrestrial net primary productivity (NPP): analysis of the seasonal atmospheric $\mathrm{CO}_{2}$ signal. Global Change Biology 5:65-76.

Nicodemus, F. E., J. C. Richmond, J. J. Hsia, I. W. Ginsberg and T. Limperis. 1977. Geometrical Considerations and Nomenclature for Reflectance. NBS Monograph 160, National Bureau of Standards, US Department of Commerce, Washington, D.C.

Painter, T. H., J. Dozier, D. A. Roberts, R. E. Davis and R. O. Green. 2003. Retrieval of subpixel snow-covered area and grain size from imaging spectrometer data. Remote Sensing of Environment 85:64-77.

Potter, C. S., S. S. Wang, N. T. Nikolov, A. D. McGuire, J. Liu, A. W. King, J. S. Kimball, R. F. Grant, S. E. Frolking, J. S. Clein, J. M. Chen and J. S. Amthor. 2001. Comparison of boreal ecosystem model sensitivity to variability in climate and forest site parameters. Journal of Geophysical Research-Atmospheres 106:33671-33687. 
Rast, M. 1999. Special Issue - ESA medium resolution imaging spectrometer (MERIS). International Journal of Remote Sensing 20:1679-1679.

Rast, M., F. Baret, M. Menenti, D. Schimel, M. M. Verstraete, W. Mauser, J. Miller and M. Schaepman. 2001. SPECTRA - Surface Processes and Ecosystem Changes Through Response Analysis. ESA, Noordwijk.

Richter, R. and D. Schlapfer. 2002. Geo-atmospheric processing of airborne imaging spectrometry data. Part 2: atmospheric/topographic correction. International Journal of Remote Sensing 23:2631-2649

Schaepman, M., S. Dangel, M. Kneubühler, D. Schlaepfer, S. Bojinski, J. Brazile, B. Kötz, G. Strub, R. Kohler, C. Popp, J. Schopfer and K. Itten. 2002. Quantitative field spectroscopic measurement instrumentation and techniques.In 1st EPFS Workshop on Field Spectrometry, NERC, Southampton, UK.

Schaepman, M., K. Itten and M. Rast. 2003a. Imaging Spectroscopy as a quantiative tool for the retrieval of biogeophysical parameters. Geographica Helvetica 2:120-130.

Schaepman, M. E. and S. Dangel. 2000. Solid laboratory calibration of a nonimaging spectroradiometer. Applied Optics 39:37543764.

Schaepman, M. E., K. I. Itten, D. Schläpfer, J. Kaiser, J. Brazile, W. Debruyn, A. Neukom, H. Feusi, P. Adolph, R. Moser, T. Schilliger, L. De Vos, G. Brandt, P. Kohler, M. Meng, J. Piesbergen, P. Strobl, J. Gavira, G. J. Ulbrich and R. Meynart. 2003b. APEX: Current status of the airborne dispersive pushbroom imaging spectrometer. In: R. Meynart, S. P. Neeck, H. Shimoda, J. B. Lurie and M. L. Aten (eds.), Sensors, Systems, and NextGeneration Satellites VII. SPIE, Barcelona. pp. 202-210.

Schlapfer, D. and R. Richter. 2002. Geo-atmospheric processing of airborne imaging spectrometry data. Part 1: parametric orthorectification. International Journal of Remote Sensing 23:26092630 .

Schoorl, J. M. and A. Veldkamp. 2001. Linking land use and landscape process modelling: a case study for the Alora region (south Spain). Agriculture Ecosystems \& Environment 85:281292.

Secker, J., K. Staenz, R. P. Gauthier and P. Budkewitsch. 2001. Vicarious calibration of airborne hyperspectral sensors in operational environments. Remote Sensing of Environment 76:81-92.

Sims, D. A. and J. A. Gamon. 2002. Relationships between leaf pigment content and spectral reflectance across a wide range of species, leaf structures and developmental stages. Remote Sensing of Environment 81:337-354.

Strub, G., M. E. Schaepman, Y. Knyazikhin and K. I. Itten. 2003. Evaluation of spectrodirectional Alfalfa canopy data acquired during DAISEX '99. Ieee Transactions on Geoscience and Remote Sensing 41:1034-1042.

Thome, K. J., S. F. Biggar and W. Wisniewski. 2003. Cross comparison of EO-1 sensors and other Earth resources sensors to Land- sat-7 ETM+ using Railroad Valley Playa. IEEE Transactions on Geoscience and Remote Sensing 41:1180-1188.

Thornton, P. E., B. E. Law, H. L. Gholz, K. L. Clark, E. Falge, D. S. Ellsworth, A. H. Golstein, R. K. Monson, D. Hollinger, M. Falk, J. Chen and J. P. Sparks. 2002. Modeling and measuring the effects of disturbance history and climate on carbon and water budgets in evergreen needleleaf forests. Agricultural and Forest Meteorology 113:185-222.

Tian, Y. H., Y. J. Wang, Y. Zhang, Y. Knyazikhin, J. Bogaert and R. B. Myneni. 2003. Radiative transfer based scaling of LAI retrievals from reflectance data of different resolutions. Remote Sensing of Environment 84:143-159.

Turner, D. P., M. Guzy, M. A. Lefsky, S. Van Tuyl, O. Sun, C. Daly and B. E. Law. 2003. Effects of land use and fine-scale environmental heterogeneity on net ecosystem production over a temperate coniferous forest landscape. Tellus Series B-Chemical and Physical Meteorology 55:657-668.

Underwood, E., S. Ustin and D. DiPietro. 2003. Mapping nonnative plants using hyperspectral imagery. Remote Sensing of Environment 86:150-161.

Valentini, R., G. Matteucci, A. J. Dolman, E. D. Schulze, C. Rebmann, E. J. Moors, A. Granier, P. Gross, N. O. Jensen, K. Pilegaard, A. Lindroth, A. Grelle, C. Bernhofer, T. Grunwald, M. Aubinet, R. Ceulemans, A. S. Kowalski, T. Vesala, U. Rannik, P. Berbigier, D. Loustau, J. Guomundsson, H. Thorgeirsson, A. Ibrom, K. Morgenstern, R. Clement, J. Moncrieff, L. Montagnani, S. Minerbi and P. G. Jarvis. 2000. Respiration as the main determinant of carbon balance in European forests. $\mathrm{Na}$ ture 404:861-865.

Verhoef, W. 1984. Light-scattering by leaf layers with application to canopy reflectance modeling - the Sail model. Remote Sensing of Environment 16:125-141.

Weiss, M., F. Baret, G. J. Smith, I. Jonckheere and P. Coppin. 2004. Review of methods for in situ leaf area index (LAI) determination Part II. Estimation of LAI, errors and sampling. Agricultural and Forest Meteorology 121:37-53.

Weiss, M., D. Troufleau, F. Baret, H. Chauki, L. Prevot, A. Olioso, N. Bruguier and N. Brisson. 2001. Coupling canopy functioning and radiative transfer models for remote sensing data assimilation. Agricultural and Forest Meteorology 108:113-128.

Wilson, K. B., D. Baldocchi, E. Falge, M. Aubinet, P. Berbigier, C. Bernhofer, H. Dolman, C. Field, A. Goldstein, A. Granier, D. Hollinger, G. Katul, B. E. Law, T. Meyers, J. Moncrieff, R. Monson, J. Tenhunen, R. Valentini, S. Verma and S. Wofsy. 2003. Diurnal centroid of ecosystem energy and carbon fluxes at FLUXNET sites. Journal of Geophysical Research-Atmospheres 108:4664.

Zarco-Tejada, P. J., J. C. Pushnik, S. Dobrowski and S. L. Ustin. 2003. Steady-state chlorophyll a fluorescence detection from canopy derivative reflectance and double-peak red-edge effects. Remote Sensing of Environment 84:283-294. 